\title{
14 MeV NEUTRON IRRADIATION EXPERIMENTS - GAMMA SPECTROSCOPY ANALYSIS AND VALIDATION AUTOMATION
}

\author{
Thomas Stainer $^{1}$, Mark R Gilbert ${ }^{1}$, Lee W Packer ${ }^{1}$, Steven Lilley $^{2}$ \\ Vignesh Gopakumar $^{1}$, and Chris Wilson ${ }^{1}$ \\ ${ }^{1}$ UK Atomic Energy Authority \\ Culham Science Centre, Abingdon, OX14 3DB, UK \\ ${ }^{2}$ ISIS Neutron and Muon Source, Science and Technology Facilities Council, \\ Rutherford Appleton Laboratory, Didcot OX11 0QX, UK \\ thomas.stainer@ukaea.uk,mark.gilbert@ukaea.uk, lee.packer@ukaea.uk, \\ steven.lilley@stfc.ac.uk, vignesh.gopakumar@ukaea.uk christopher.wilson@ukaea.uk
}

\begin{abstract}
An important area of research required for fusion reactor design is the study of materials under high energy neutron irradiation. Deuterium-Tritium (D-T) reactions release 14.1 $\mathrm{MeV}$ neutrons and material studies of such high energy neutrons focusing on transmutation and activation are paramount for fusion tokamak devices such as ITER and DEMO. In order to understand neutron damage and transmutation-induced radioactivity in fusion regime energies, a series of experimental campaigns were performed at the ASP facility based at Aldermaston in the UK, which uses a deuteron accelerator to bombard a tritiumloaded target and generate $14 \mathrm{MeV}$-neutron emission rates of up to $2.5 \times 10^{11} \mathrm{~s}^{-1}$. In this work, a holistic treatment of the 11,000 gamma spectra (time series data) collected over five experimental campaigns is applied to identify radioisotopes and validate nuclear data and the inventory code, FISPACT-II. Whilst previous analysis has examined single spectra and foil irradiation's using traditional, human-driven methods, this work applies novel methods using Artificial Neural Networks (ANN) and classification algorithms to allow a fully automated approach. Using such methods we show good broad agreement with FISPACT-II inventory simulations, and an overview of results are given as $\mathrm{C} / \mathrm{E}$ values.
\end{abstract}

KEYWORDS: ASP, FISPACT-II, Neural Network

\section{INTRODUCTION}

Designing tokamak devices such as ITER and DEMO requires in-depth knowledge and understanding of material damage, transmutation and activation due to $14.1 \mathrm{MeV}$ neutrons, arising from Deuterium-Tritium (D-T) reactions. These reactors expect to experience neutron fluxes of the order of $10^{18}$ neutrons $\mathrm{m}^{-2} \mathrm{~s}^{-1}$, and quantities such as nuclear heating, neutron damage and transmutation-induced radioactivity are crucial for their design and operation [1]. Currently, no facilities exist which offer the ability to expose materials to neutron fluxes and energies equivalent to those predicted for fusion. Whilst plans are underway to develop a DEMO Oriented Neutron Source (DONES) [2] to tackle this issue, very little data is available for material exposure under 14 $\mathrm{MeV}$ neutron irradiation. In addition to material damage, gamma spectroscopy offers the ability to 
monitor the plasma inside the reactor through the determination of neutron fluences in such intense and harsh environments.

The multi-physics and inventory code FISPACT-II [3] allows time dimensional analysis of neutron irradiated materials, based on an extensive set of modern nuclear data libraries. Codes such as FISPACT-II are crucial when developing fusion nuclear technology, but are highly reliant on accurate and complete nuclear data libraries. Spectral data is included in the nuclear data libraries and FISPACT-II is capable of reading spectral lines and can appropriately produce discrete spectra based on radioisotopes present in the inventory. This offers a direct approach to compare and validate existing nuclear data libraries with experimental data in the fusion energy regime [4] [5]. This work then presents an automated approach to perform direct comparison and validation between ASP gamma spectra data and inventory simulations for a wide range of materials and reactions.

\section{ASP FACILITY}

The ASP facility based at Aldermaston in the UK, generates deuterium-tritium neutrons via a low-energy high-current deuteron accelerator, operating at $50 \mathrm{kV}$ extract potential, focused onto a tritium target [6]. Prior to impact on the target the ionised deuterium beam is focused and collimated along the 18 meter accelerator tube and assembly, and is focused to a diameter of roughly 1 $\mathrm{cm}$. The subsequent fusion reaction between deuterium and tritium then produces neutrons at an emission rate of up to $2.5 \times 10^{11} \mathrm{~s}^{-1}$. Thin film foils consisting of a variety of materials are placed directly in front of the target for an irradiation period and promptly extracted via the specially adapted pneumatic rabbit system. The rabbit system can perform the extraction to the measurement area in around 10 seconds. Gamma spectroscopy measurements are then performed using a High-Purity germanium (HPGe) gamma-ray spectrometer to measure the decay emission energy spectrum.

\subsection{Modelling}

A Computer-Aided Design (CAD) model has been constructed for the accelerator assembly [7]. A simplified version of this model is then used with MCNP6 [8] to estimate the surface average fluence at various distances from the target, and provide an expected neutron spectrum at the target. A near isotropic source definition used in the model derives from a basic kinetic model and models incident neutrons near the target with binned energy and angular distributions. The estimated incident particle energy spectrum at the foil surface has been determined with and without the rabbit system, using FENDL 3.1 [9] and ENDF/B.VII [10] nuclear data libraries.

A Geant4 [11] and MCNP model was used to estimate gamma-ray detector efficiencies based on the Monte Carlo method, matching incident peak energies with energy deposited. The latter was used to match experimental calibration data at the time of the experiments, with the former being used for comparison and uncertainty estimates. It was assumed that foils are placed directly in contact with the top of the detector end cap, however, this was not always strictly the case. The foil to end cup distance is estimated at $1 \mathrm{~cm}$, with values taken $1 \mathrm{~cm}$ either side to provide a conservative estimate of the uncertainty due to the source position. An exponential fit of the form $\exp \sum_{i=0}^{5} a_{i} \ln ^{i}\left(E / E_{\text {max }}\right)$, with $E_{\text {max }}=e^{2} \sim 7.39 \mathrm{MeV}$ aligned well with the data points. Since 
the MCNP fit has been well adapted (refinements mainly to the dead layer) specifically to match with experimentally collected data, the fit is used to estimate detector efficiencies in this work.

\subsection{Experimental Campaigns}

Five experimental campaigns represent a series of five individual trips to Aldermaston, with each consisting of an itinerary of foils to be irradiated. Campaign 1 involves only single foil irradiation's, with ${ }^{238}$ U-based fission counters used to monitor neutron fluences. The fission counters were placed on either side of the target. Spatial fluctuations of the beam can dramatically affect results from the fission counters and therefore provide an unreliable estimate of the neutron fluence. Due to this, campaign 1 is excluded from this work. Further campaigns (2, 3, 4, and 5) then mediate this issue by using reference $\mathrm{Al}$ and $\mathrm{Fe}$ foils in unison with target foils to determine neutron fluences, via well-defined reactions as shown in table 1. Small-sized foils, all of diameter $12 \mathrm{~mm}$, are used to negate spatial variations of the beam, and thin foils of thicknesses from 0.1 to $0.5 \mathrm{~mm}$, then minimise self-shielding effects and attenuation to other adjacent foils in the sample. The overview of the 211 foils used in 168 experiments with their associated irradiation and count times are shown in figure 1.

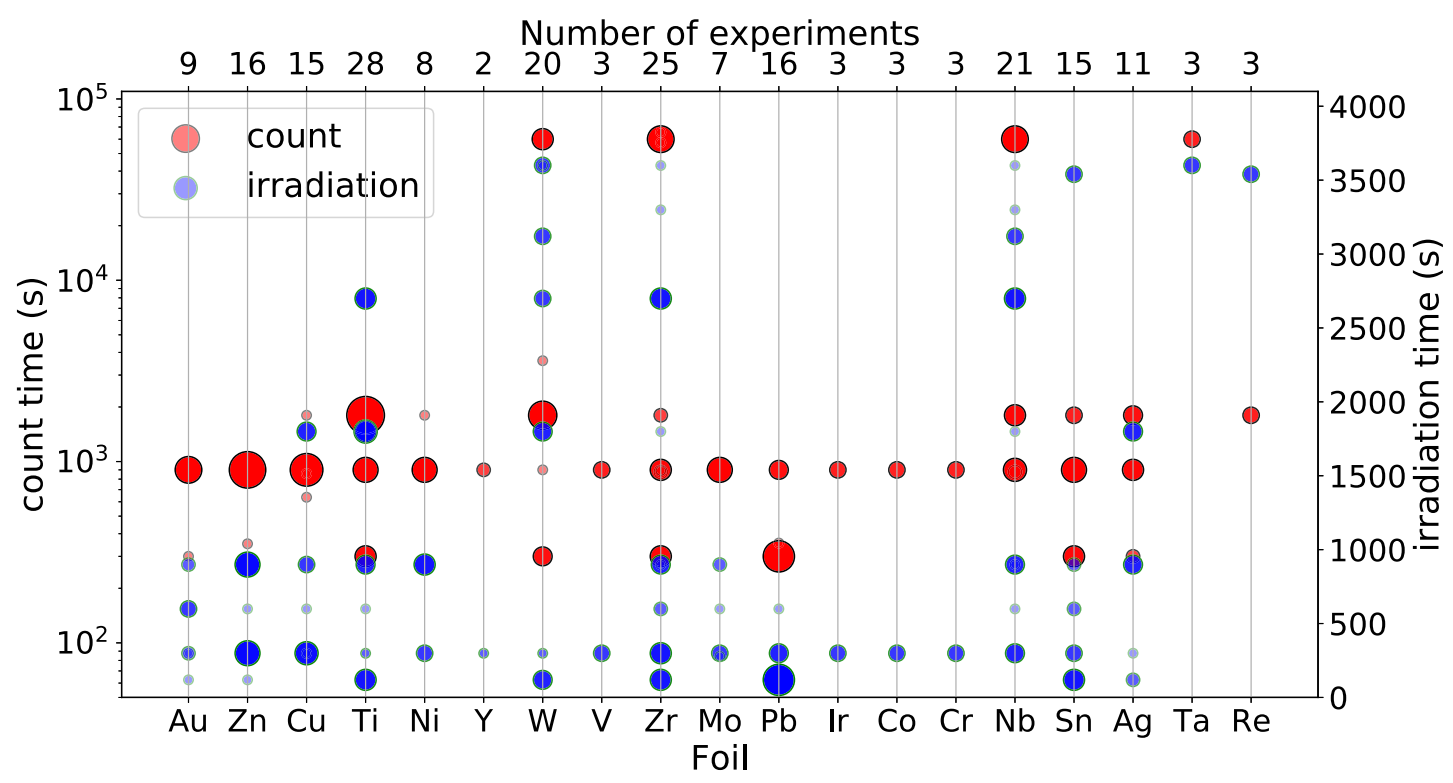

Figure 1: The foils used in experimental campaigns 2, 3, 4, and 5, with their corresponding irradiation (blue, right linear y-axis) and count (red, left logarithmic y-axis) times. The diameter of the point represents the number of repeated experiments (same foil and irradiation/cooling times). The full data set covers 211 experiments with individual foil experiment counts listed along the top $x$-axis. Reference foils are excluded.

Radionuclides produced from $\mathrm{Al}$ and $\mathrm{Fe}$ reactions benefit from having half-lives comparable to laboratory transfer and measurement times at $9.458 \pm 0.012$ minutes, $2.5824 \pm 0.0048$ hours, and $14.957 \pm 0.002$ hours for ${ }^{27} \mathrm{Mg},{ }^{56} \mathrm{Mn}$, and ${ }^{24} \mathrm{Na}$ respectively. Spectral line identification becomes slightly problematic with ${ }^{27} \mathrm{Mg}$ and ${ }^{56} \mathrm{Mn}$ each having a line at an energy in close proximity to the 


\begin{tabular}{|l|l|l|l|}
\hline Reaction pathway & Radioisotope & Gamma energy $(\mathbf{M e V})$ & Gamma intensity \\
\hline${ }^{27} \mathrm{Al}(\mathrm{n}, \mathrm{p})$ & ${ }^{27} \mathrm{Mg}$ & $0.843700 \pm 0.000100$ & $0.720000 \pm 0.010000$ \\
${ }^{56} \mathrm{Fe}(\mathrm{n}, \mathrm{p})$ & ${ }^{56} \mathrm{Mn}$ & $0.846764 \pm 0.000001$ & $0.988300 \pm 0.000300$ \\
${ }^{27} \mathrm{Al}(\mathrm{n}, \mathrm{p})$ & ${ }^{27} \mathrm{Mg}$ & $1.014400 \pm 0.000100$ & $0.282000 \pm 0.010000$ \\
${ }^{27} \mathrm{Al}(\mathrm{n}, \alpha)$ & ${ }^{24} \mathrm{Na}$ & $1.368630 \pm 0.000005$ & $0.999935 \pm 0.000005$ \\
${ }^{56} \mathrm{Fe}(\mathrm{n}, \mathrm{p})$ & ${ }^{56} \mathrm{Mn}$ & $1.810730 \pm 0.000004$ & $0.276000 \pm 0.006000$ \\
${ }^{56} \mathrm{Fe}(\mathrm{n}, \mathrm{p})$ & ${ }^{56} \mathrm{Mn}$ & $2.113090 \pm 0.000006$ & $0.148000 \pm 0.004000$ \\
${ }^{56} \mathrm{Fe}(\mathrm{n}, \mathrm{p})$ & ${ }^{56} \mathrm{Mn}$ & $2.523060 \pm 0.000050$ & $0.013000 \pm 0.000300$ \\
${ }^{27} \mathrm{Al}(\mathrm{n}, \alpha)$ & ${ }^{24} \mathrm{Na}$ & $2.754010 \pm 0.000011$ & $0.998720 \pm 0.000080$ \\
\hline
\end{tabular}

Table 1: The list of reactions and the radionuclides produced for the Aluminum and Iron foils which are used to estimate neutron fluence. Each radioisotopes dominant $(\geq 1 \%$ intensity) gamma decay energies and intensities are shown in the table. Values and uncertainties are based on JEFF 3.3 data values [12][13][14] released with FISPACT-II version 4.0.

other (around $0.84 \mathrm{MeV}$ ). However it was possible to discriminate between the peaks using the gamma-ray spectrometer employed in this work.

\section{PEAK IDENTIFICATION}

Traditionally, peak searching algorithms perform moving averages over sliding windows, with additional techniques taking into account first and second derivatives [15] [16] [17]. In our approach, algorithms existing in open source tools and packages, such as ROOT [18], were assessed and combined with these traditional methods. Most of these methods require some prior knowledge of where to expect peaks, or for agnostic procedures, fail to identify peaks in close proximity, known as multiplets, as is the case for ${ }^{27} \mathrm{Al}(\mathrm{n}, \mathrm{p}){ }^{27} \mathrm{Mg}$, and ${ }^{56} \mathrm{Fe}(\mathrm{n}, \mathrm{p}){ }^{56} \mathrm{Mn}$ reactions. Additional difficulties arise when attempting to identify peaks in regions governed by low statistics and counts varying dramatically across a log scale. We examine the use of supervised learning techniques using Artificial Neutral Networks (ANNs) for peak classification [19][20] for comparison.

With 16384 channels across the HPGe detector the required network and size of the input layer to the network is too vast to computationally realise. Instead a discretized bin method was used, whereby the energy regime was fragmented into equal sizes of $N$ bins, where $N \in\{5,7,9,11,13$, $15,17,19,21\}$. Each ANN was trained on real and synthetically generated data sets, based on the campaign data and a Geant4 detector model, using labels from simulation and through use of other conventional peak finding algorithms. The training data sets consist of over 200,000 samples, with roughly equal proportions of each label (peak or no peak). The networks consisted of 6 hidden layers, with the input layer consisting of $N$ perceptrons and values log normalised. The output layer of size 1 represents the probability of a peak, as in figure 2 .

A more traditional method of peak finding, using a moving average window taking into account 


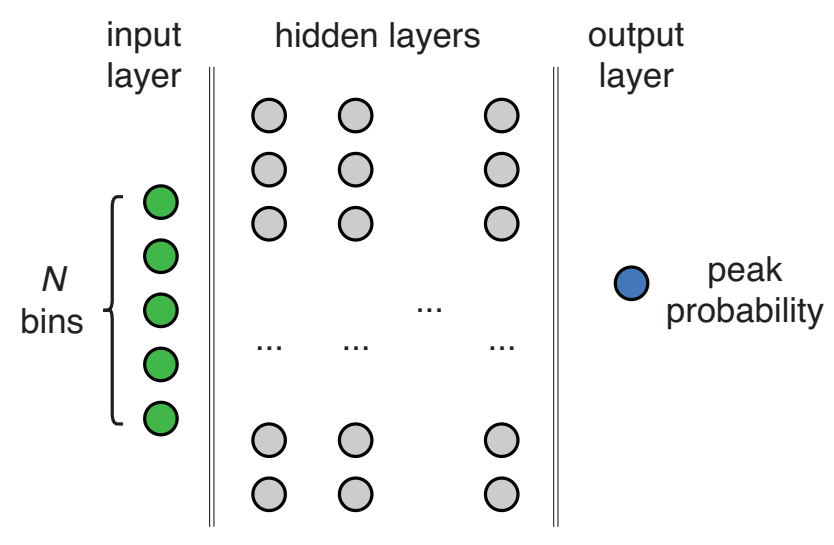

Figure 2: The architecture of the neural network used for peak finding. It consists of $N$ input nodes varying between 5 to 21 in odd numbers (so the peak is always at the centre index), 6 hidden layers of varying number of perceptrons, and a single output perceptron indicating the peak probability.

standard deviation thresholds and examining first and second derivatives is used for comparison. Using a windows size of 40 and threshold of mean plus sigma threshold of 2 was determined to provide the optimal parameters for this method, given constraints on maximising true positives and minimising false-positives.
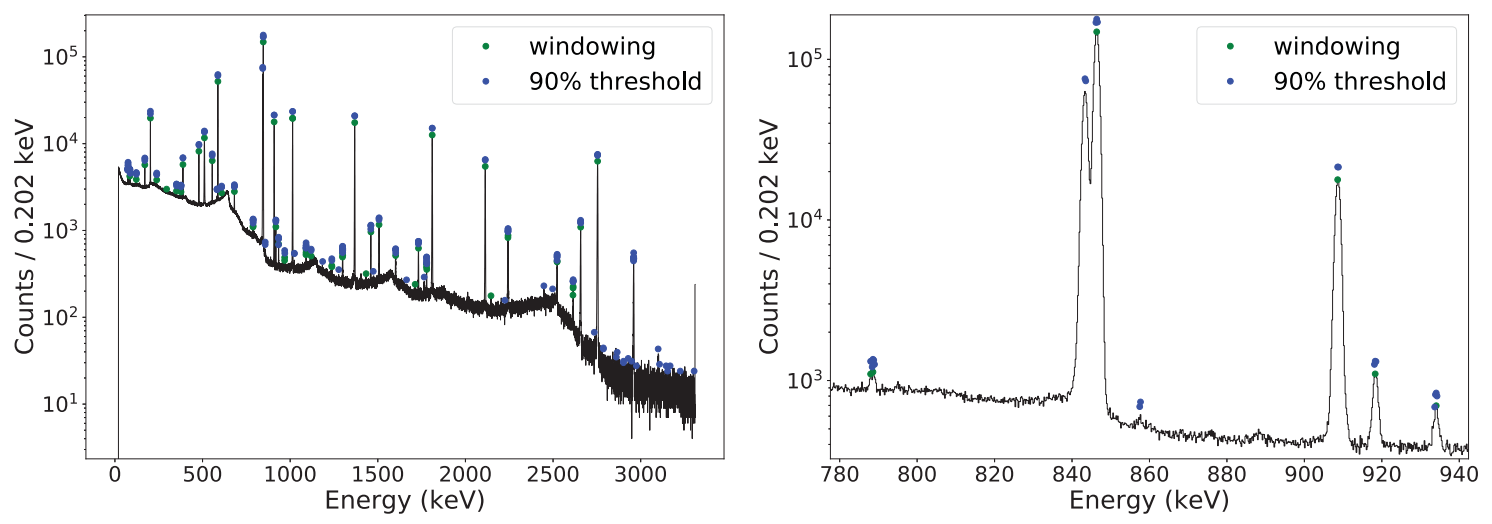

Figure 3: The recorded gamma spectrum for a $\mathrm{Zr}$ foil experiment at a count time of $64.5 \mathrm{~s}$ following an irradiation for $299 \mathrm{~s}$. Peaks found with the conventional window method using a moving average of 40 points and 2 sigma threshold are shown in green, and the ANN with

$N=13$ bins and a peak probability threshold of $90 \%$ is shown in blue. Blue points are shifted vertically for visualisation purposes only, to avoid overlapping of points. Left shows full energy range, with the right showing a shorter energy range to highlight the duplet near $843 \mathrm{keV}$.

The ANN shows good agreement with conventional methods and surpasses it for multiplet recognition, as can be seen in figure 3. It is stressed that both methods required no prior knowledge of the 
peaks and did not enforce any requirements on the peak. No smoothing or background subtraction was applied, to avoid loss of peak information. Considering this, the ANN produces remarkable results and can correctly identify the duplet for ${ }^{27} \mathrm{Mg}$ and ${ }^{56} \mathrm{Mn}$ at around 843 and $846 \mathrm{keV}$, which is missed by conventional methods. It is also noticeable that for the experiment presented in the figures, and in general, the ANNs produces a larger number of false-positives in the high energy range when counts are below 500. This varies depending on the number of input nodes, $N$, but is likely due to a lack of accurately labeled data in this regime.

\section{FLUX ESTIMATION}

The reference peaks in table 1 are used to estimate the incident neutron flux per each experiment. Due to poor statistics, low intensities and efficiencies, and long half-life of ${ }^{24} \mathrm{Na}$ we choose to ignore these peaks and base estimates on Gaussian fits to peaks at 843, 846, 1014, and $1810 \mathrm{keV}$. The SNIP method [21] is used to estimate and remove background counts, line efficiencies and detector efficiencies are both taken into account. Thus the activity at the end of the irradiation, $A_{0}$, can be determined based on an exponential fit of integral counts with real time.

The flux, $\phi$, is then related to the activity via equation 1 [22]. With $\alpha_{R R}$ representing the total reaction rate, $N_{p}$ as the number of parent particles, $\lambda$ as the decay constant of that nuclide, and $t_{\text {irrad }}$ as the irradiation time. Reaction rates are determined from FISPACT-II version 4.0 using TENDL 2017 [23] libraries.

$$
\phi=\frac{A_{0}}{\alpha_{R R} N_{p}\left(1-e^{\left.-\lambda t_{\text {irrad }}\right)}\right.}
$$

The calculated fluxes for each experiment are shown in figure 4, showing values in the region of $10^{8}$ to $10^{9}$ neutrons $\mathrm{cm}^{-2} \mathrm{~s}^{-1}$. It is obvious from this figure that the flux decreases with time, starting with a mean flux of $(8.5 \pm 1.9) \times 10^{8}$ for campaign 2 , reducing to $(5.4 \pm 1.5) \times 10^{8}$ and $(3.4 \pm 1.1) \times 10^{8}$ for campaigns 3 and 5 respectively. Whilst there is no record of changes to the target it cannot be ruled out. An alternative theory for the decrease may have been due to tritium depletion of the beam target. The beam was indeed moved between experiments to reduce such effects, which also introduces wide variation in flux estimates within campaigns.

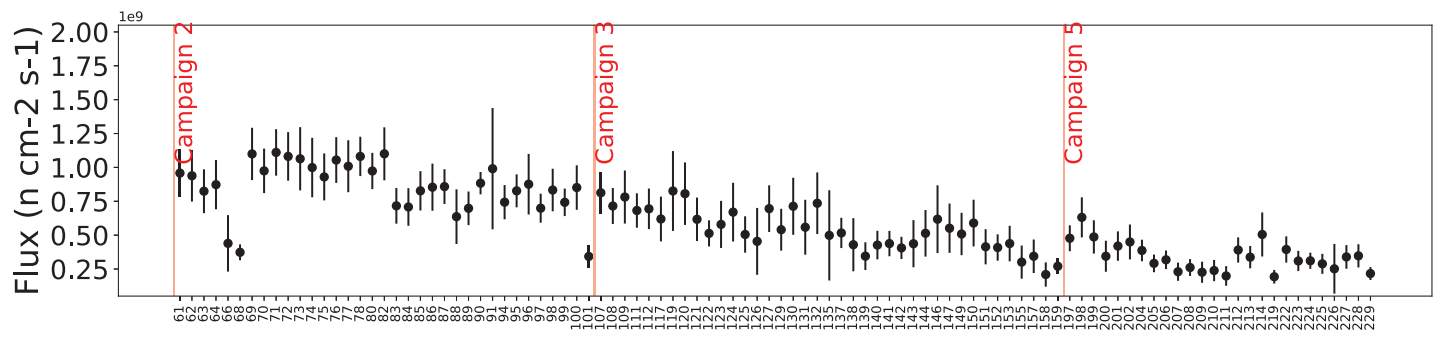

Figure 4: The flux estimates (neutrons $\mathrm{cm}^{-2} \mathrm{~s}^{-1}$ ) for each experiment based on exponential fits to gamma lines from reference foils in table 1. Campaign 4 yielded no conclusive fits for flux estimates and are excluded. 


\section{COMPARISON WITH FISPACT-II}

An automated system has been constructed in order to form a digital twin of each experiment, using FISPACT-II. UKAEA has developed an internal version of FISPACT-II which allows it to be driven via an Application Programming Interface (API). Besides the several performance advantages which makes automation of large data sets now possible, it allows trivial interfacing with other codes and allows such holistic studies to be realised. The FISPACT-II API allows direct access to nuclear data lines based on current inventories and allows bespoke gamma bounds to match that of a HPGe detector with 16384 channels. It is then trivial to compare simulation results with experiment following the integration of gamma lines to match experiment measurement times.
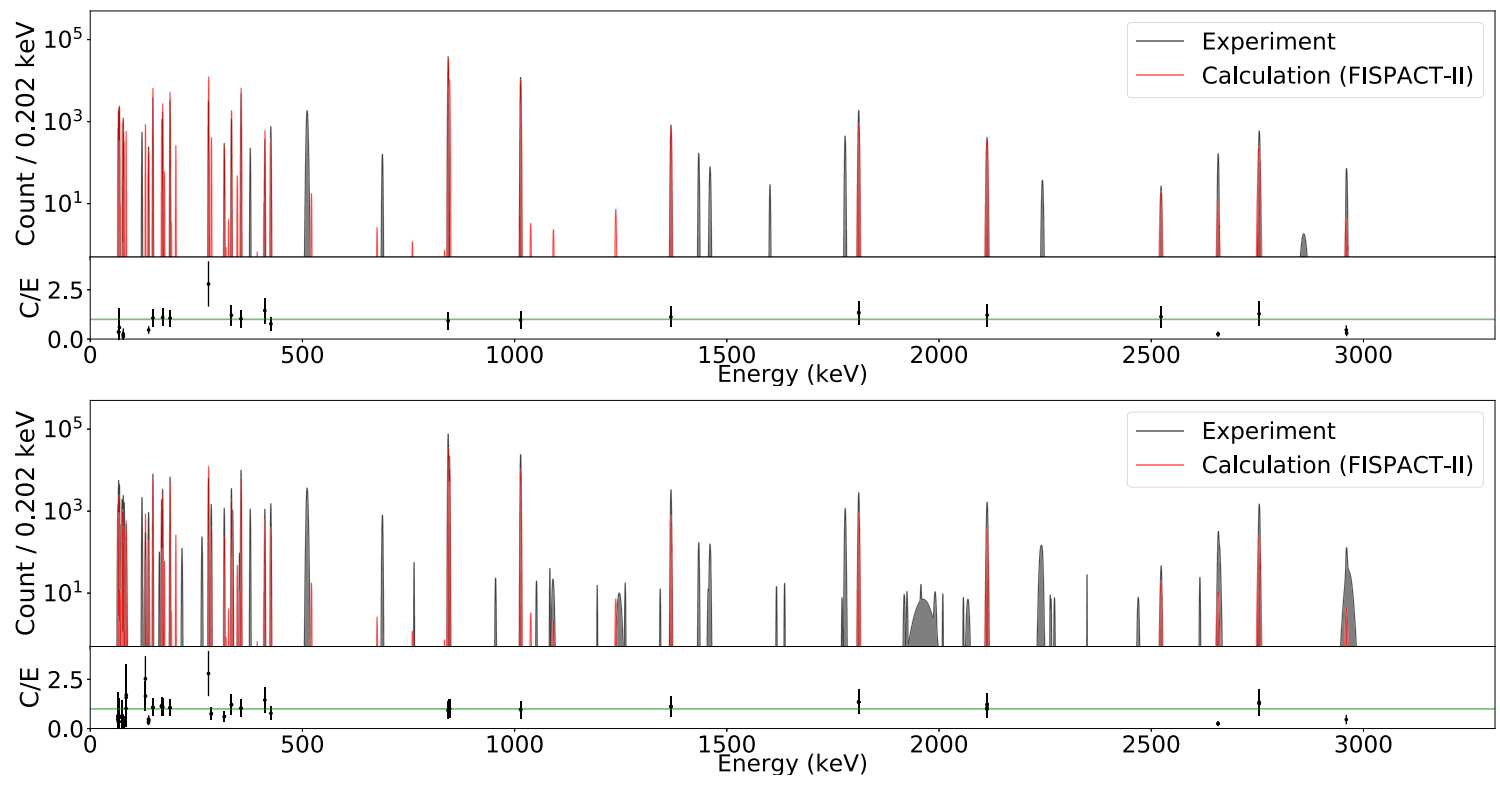

Figure 5: The comparison of FISPACT-II simulated gamma lines (red) to experimental gamma lines (black) following peak identification, background subtraction and Gaussian broadening using a sliding window approach (top) and a neural network (bottom) for peak identification, for the full energy range. $\mathrm{C} / \mathrm{E}$ values are given below each spectrum.

All experimental data is stored using a NoSQL database (MongoDB) and each experiment is analysed by querying the database and constructing an equivalent FISPACT-II simulation. Peak matching and background removal algorithms are performed on the data, as described previously, and selected peaks are fitted with Gaussian distributions which are matched to broadened FISPACT-II lines, taking into account detector efficiencies. This comparison is shown for a Au experiment (experiment 61) at 924 seconds (final measurement time) following irradiation in figure 5 using $\mathrm{C} / \mathrm{E}$ values. The FISPACT-II simulation line count is given as $\mathrm{C}$, divided by the experimental data line count estimation, given as $\mathrm{E}$, for each peak.

The combined C/E results for all experiments are binned into a histogram per matched peak and shown in figure 7 . The results from traditional window method and the ANN are compared. The clear outliers near $\mathrm{C} / \mathrm{E}=0$ in the first bin show the simulation is severely under predicting peaks for experiments containing multiple foils (more than 4) or falsely matching the $511 \mathrm{keV}$ positron- 

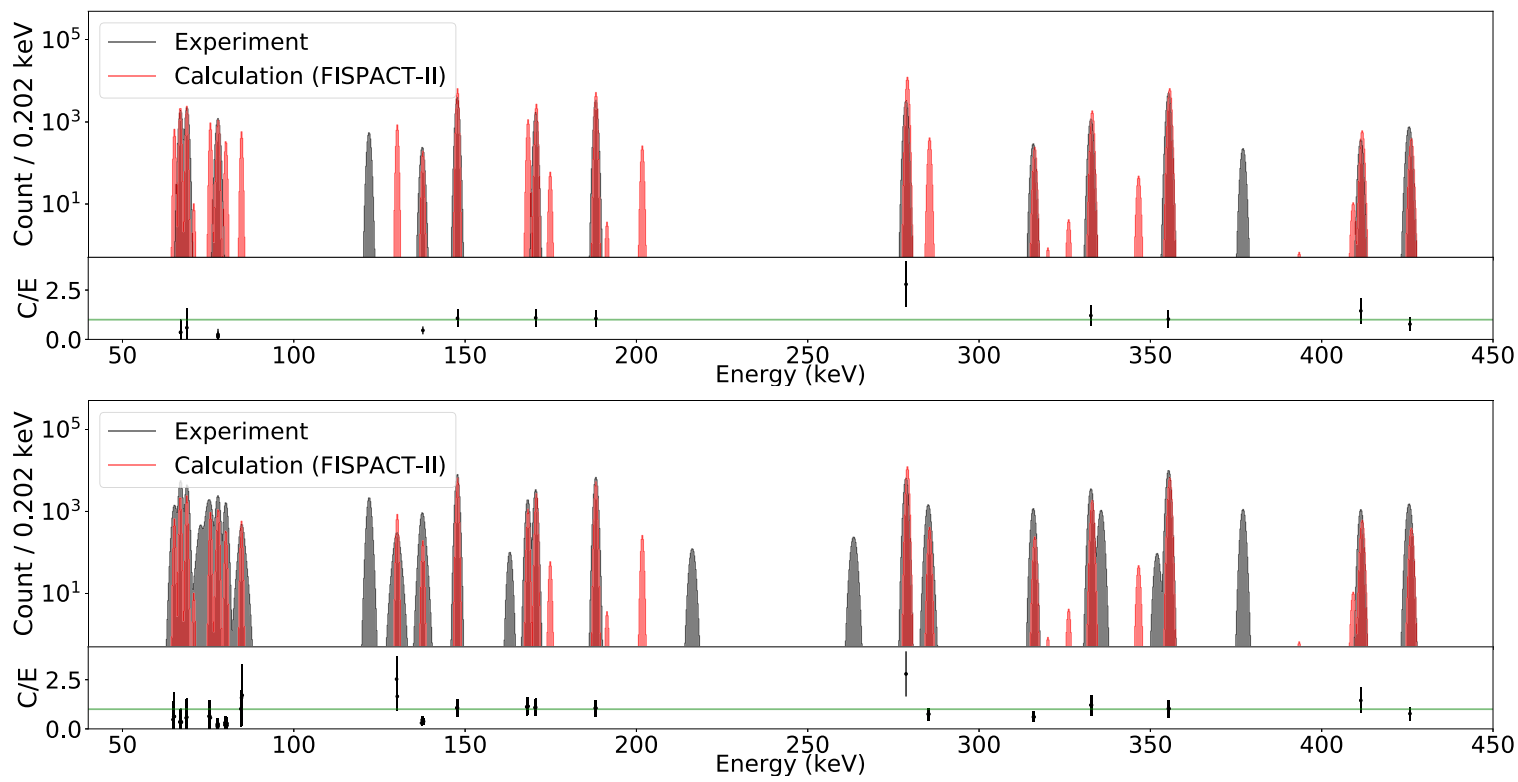

Figure 6: The comparison of FISPACT-II simulated gamma lines (red) to experimental gamma lines (black) following peak identification, background subtraction and Gaussian broadening using a sliding window approach (top) and a neural network (bottom) for peak identification, in the reduced energy range $50 \mathrm{keV}$ to $450 \mathrm{keV}$.

electron annihilation peak. In the case of the latter FISPACT-II identifies spectral lines that are very close to the signature peak at much lower intensity causing a low $\mathrm{C} / \mathrm{E}$ value. This is much more evident for the ANN, due to the larger number of peaks detected. Ignoring the outliers it is evident that generally there is good agreement with most peaks, centred around 1 , however a slight skew towards $\mathrm{C}<\mathrm{E}$ is noticeable, particularly in the low energy regime $(<500 \mathrm{keV})$. The peaks identified with the ANN show a much larger number agreeing with calculation and altogether show a better general agreement.

\section{CONCLUSIONS}

An automated approach to peak identification can save considerable time in gamma spectroscopy, which is typically performed by hand at the cost of prolonged human effort. Along with traditional windowing methods, neural networks show promising results which can dramatically improve the number of peaks correctly identified, specifically for multiplets, but also introduce high falsepositive rates, typically in higher energy and low count regimes. Much work is still needed to develop these methods further and hybrid approaches can be adopted in the future to improve multiplets whilst reducing false-positive rates.

It can be seen that whilst there is some good agreement between simulation and experiment, wide gaps remain. It is hard to conclude and comment on the direct source of disagreement, better understanding and propagation of uncertainties is needed in modelling.

This holistic approach has shown the potential that this process can be fully automated and can 

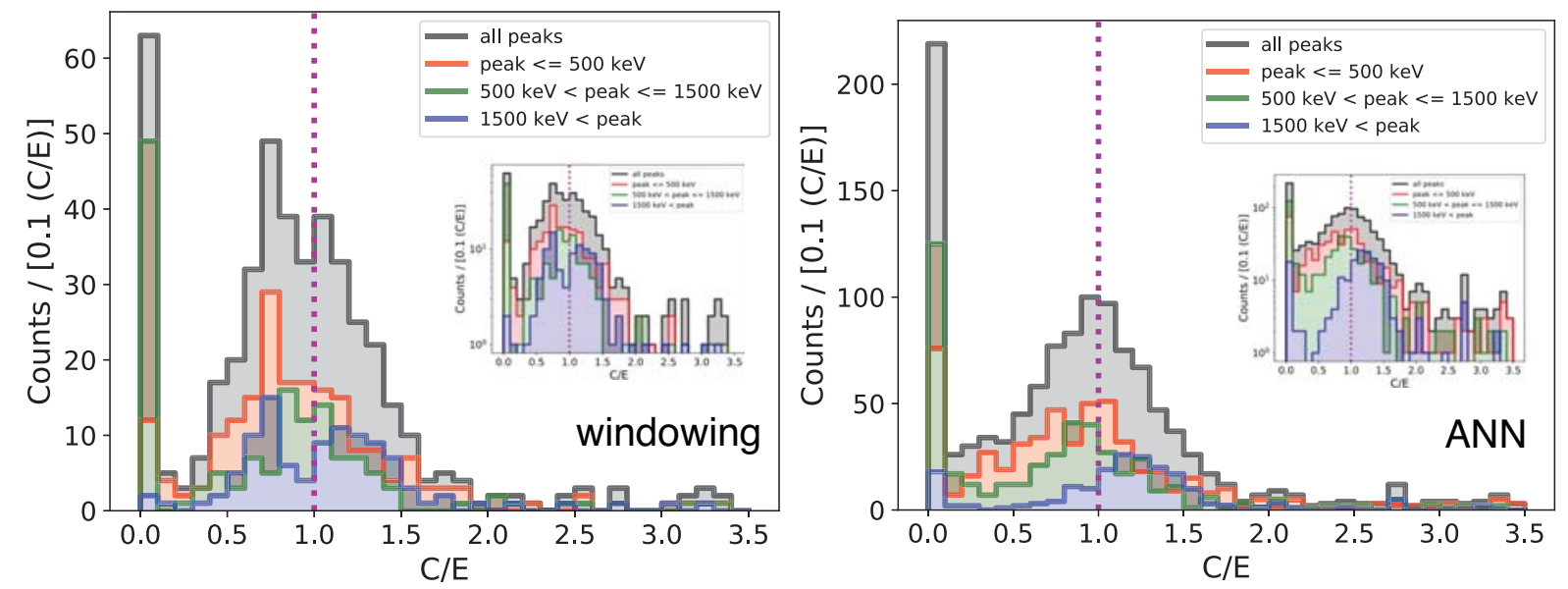

Figure 7: The distribution of FISPACT-II calculated peak values (C) divided by experimental peak values ( $E$ ) for matched peaks using windowing method (left) and ANN (right) across all valid experiments. Equivalent $y$-axis log scaled versions of each plot are inserted for comparison.

be used to perform analysis immediately after data collection, providing feedback and data quality assessments to experimentalists whilst on site. Therefore allowing data to be analysed and experiments to be altered and adjusted within timescales of hours instead of years.

\section{ACKNOWLEDGEMENTS}

This work was funded by the RCUK Energy Programme [grant number EP/T012250/1]. To obtain further information on the data and models underlying this paper please contact publicationsmanager@ukaea.uk.

\section{REFERENCES}

[1] G. Federici, W. Biel, M. Gilbert, R. Kemp, N. Taylor, and R. Wenninger. "European DEMO design strategy and consequences for materials." Nuclear Fusion, volume 57(9) (2017).

[2] A. Ibarra and et. al. "The IFMIF-DONES Project: preliminary engineering design." Nuclear Fusion, volume 58(10) (2018).

[3] J.-C. Sublet, J. Eastwood, J. Morgan, M. Gilbert, M. Fleming, and W. Arter. "FISPACTII: An Advanced Simulation System for Activation, Transmutation and Material Modelling." Nuclear Data Sheets, volume 139, pp. 77 - 137 (2017).

[4] L. Packer, M. Gilbert, and S. Lilley. "Integral Cross Section Measurements Around $14 \mathrm{MeV}$ for Validation of Activation Libraries." Nuclear Data Sheets, volume 119, pp. 173 - 175 (2014).

[5] L. Packer, S. Hughes, M. Gilbert, S. Lilley, and R. Pampin. "Recent integral cross section validation measurements at the ASP facility." Fusion Engineering and Design, volume 88(9), pp. $2617-2620$ (2013). 
[6] AWE. "Discovery, Issue 9." The Science \& Technology Journal of AWE (2004).

[7] J. Pottage. "ASP Facility MCNP Model.” (2010). Personal communication.

[8] C. J. W. et al. "MCNP6.2 Release Notes." report la-ur-18-20808, Los Alamos National Laboratory (2018).

[9] D. W. Muir, S. Ganesan, and A. B. Pashchenko. "FENDL: A Reference Nuclear Data Library for Fusion Applications." Nuclear Data for Science and Technology Research Reports in Physics Springer, Berlin, Heidelberg (1992).

[10] D. A. Brown and et al. "ENDF/B-VIII.0: The 8th Major Release of the Nuclear Reaction Data Library with CIELO-project Cross Sections, New Standards and Thermal Scattering Data." Nuclear Data Sheets, volume 148, pp. 1 - 142 (2018). Special Issue on Nuclear Reaction Data.

[11] S. Agostinelli and et al. "Geant4 - a simulation toolkit." Nuclear Instruments and Methods in Physics Research Section A: Accelerators, Spectrometers, Detectors and Associated Equipment, volume 506(3), pp. 250 - 303 (2003).

[12] R. Bigoni, S. Bloom, and K. Tirsell. "Beta-gamma (CP) correlation and gamma spectroscopy in 27Mg." Nuclear Physics A, volume 134(3), pp. 620 - 632 (1969).

[13] P. Endt and C. V. D. Leun. "Energy levels of A = 2144 nuclei (VI)." Nuclear Physics A, volume 310(1), pp. 1 - 751 (1978).

[14] A. Sher and B. Pate. "The decay of 56Co and 56Mn." Nuclear Physics A, volume 112(1), pp. $85-96$ (1968).

[15] J. Routti and S. G. Prussin. "Photopeak method for the computer analysis of gamma-ray spectra from semiconductor detectors." Nuc Instr and Methods, pp. 125-142 (1969).

[16] M. Mariscotti. "A method for automatic identification of peaks in the presence of background and its application to spectrum analysis." Nuclear Instruments and Methods, volume 50, pp. 309-320 (1967).

[17] P. Yazid. "Y-Spect: A Multi-Method Gamma Spectrometry Analysis Program." Atom Indonesia, volume 39, pp. 40-49 (2013).

[18] R. Brun and F. Rademakers. "ROOT - An Object Oriented Data Analysis Framework." Nucl Inst \& Meth in Phys Res, volume 389, pp. 81 -86 (1997).

[19] P. Keller and et al. "Nuclear Spectral Analysis via Artificial Neural Networks for Waste Handling." IEEE Transactions on Nuclear Science, volume 42 (1995).

[20] S. Murray and et al. "A Low Complexity Radioisotope Identification System Using an Integrated Multichannel Analyzer and Embedded Neural Network." IEEE International Symposium on Circuits and Systems, pp. 1-5 (2019).

[21] C. Ryan, E. Clayton, W. Griffin, S. Sie, and D. Cousens. "SNIP, a statistics-sensitive background treatment for the quantitative analysis of PIXE spectra in geoscience applications." Nuclear Instruments and Methods in Physics Research Section B: Beam Interactions with Materials and Atoms, volume 34(3), pp. 396 - 402 (1988).

[22] K. S. Krane. Introductory Nuclear Physics. John Wiley \& Sons, New York, US (1988).

[23] A. Koning and D. Rochman. "Modern Nuclear Data Evaluation with the TALYS Code System." Nuclear Data Sheets, volume 113(12), pp. 2841 - 2934 (2012). Special Issue on Nuclear Reaction Data. 